\title{
Posterior talar process as a suitable cell source for treatment of cartilage and osteochondral defects of the talus
}

\author{
S. I. Correia ${ }^{1,2}$, J. Silva-Correia ${ }^{1,2}$, H. Pereira ${ }^{1,2,3,4}$, R. F. Canadas ${ }^{1,2}$, A. da Silva Morais $^{1,2}$, A. M. Frias ${ }^{1,5}$, \\ R. A. Sousa ${ }^{1,5}$, C. N. van Dijk ${ }^{3,6}$, J. Espregueira-Mendes ${ }^{1,2,3}$, R. L. Reis ${ }^{1,2}$ and J. M. Oliveira ${ }^{1,2 *}$ \\ ${ }^{1} 3 B s$ Research Group, Biomaterials, Biodegradables and Biomimetics, University of Minho, Headquarters of the European Institute of \\ Excellence on Tissue Engineering and Regenerative Medicine, AvePark 4805-017, Barco, Guimarães, Portugal \\ ${ }^{2}$ ICVS/3Bs, PT Government Associate Laboratory, Braga/Guimarães, Portugal \\ ${ }^{3}$ Clínica do Dragão - Espregueira-Mendes Sports Centre - FIFA Medical Centre of Excellence, F.C. Porto Stadium, Minho University and \\ Porto University Research Centre, Portugal \\ ${ }^{4}$ Orthopaedic Department Centro Hospitalar Póvoa de Varzim, Vila do Conde, Portugal \\ ${ }^{5}$ Stemmatters, Biotecnologia e Medicina Regenerativa SA, 4805-017, Guimarães, Portugal \\ ${ }^{6}$ Orthopaedic Department, Amsterdam Medical Centre, The Netherlands
}

\begin{abstract}
Osteochondral defects of the ankle are common lesions affecting the talar cartilage and subchondral bone. Current treatments include cell-based therapies but are frequently associated with donor-site morbidity. Our objective is to characterize the posterior process of the talus (SP) and the os trigonum (OT) tissues and investigate their potential as a new source of viable cells for application in tissue engineering and regenerative medicine. SP and OT tissues obtained from six patients were characterized by micro-computed tomography and histological, histomorphometric and immunohistochemical analyses. Proliferation and viability of isolated cells were evaluated by MTS assay, DNA quantification and live/dead staining. The TUNEL assay was performed to evaluate cell death by apoptosis. Moreover, the production of extracellular matrix was evaluated by toluidine blue staining, whereas cells phenotype was investigated by flow cytometry. Characterization of ankle explants showed the presence of a cartilage tissue layer in both SP and OT tissues, which represented at least $20 \%$, on average, of the explant. The presence of type II collagen was detected in the extracellular matrix. Isolated cells presented a round morphology typical of chondrocytes. In in vitro studies, cells were viable and proliferating for up to 21 days of culture. No signs of apoptosis were detected. Flowcytometry analysis revealed that isolated cells maintained the expression of several chondrocytic markers during culture. The results indicated that the SP and OT tissues were a reliable source of viable chondrocytes, which could find promising applications in ACI/MACI strategies with minimal concerns regarding donor zone complications. Copyright (c) 2015 John Wiley \& Sons, Ltd.
\end{abstract}

Received 26 June 2015; Accepted 9 September 2015

Keywords ankle; osteochondral lesions; os trigonum; regenerative medicine; Stieda process; talus lesions

\footnotetext{
*Correspondence to: Joaquim M. Oliveira, 3Bs Research Group, Biomaterials, Biodegradables and Biomimetics, University of Minho, Headquarters of the European Institute of Excellence on Tissue Engineering and Regenerative Medicine, AvePark, Parque de Ciência e Tecnologia, Zona Industrial da Gandra, 4805-017 Barco GMR, Portugal. E-mail: miguel.oliveira@dep. uminho.pt
}

\section{Introduction}

Osteochondral defects (OCDs) of the ankle represent 4\% of all osteochondral lesions in the body (Reddy et al., 2007). Ankle OCDs affecting both the talar cartilage and subchondral bone are mostly caused by single or multiple traumatic events. These types of lesions frequently progress to partial or complete detachment of the fragment, causing deep ankle pain, especially if associated with 
weight bearing (Qiu et al., 2003). Impaired function, limited range of motion, stiffness, catching, locking and swelling have been described as the most significant associated problems (van Dijk et al., 2010; Zengerink et al., 2010).

Although some OCDs of the ankle are asymptomatic, these can progress to cartilage degeneration and osteoarthritis, thus being a major concern in orthopaedics (Schachter et al., 2005). In this case, conservative treatment is restricted to stable defects in young patients (Anders et al., 2012). In turn, symptomatic OCDs that extend to bone often require surgical treatment in order to restore physiological joint function and prevent the progression of secondary degeneration (O’Driscoll, 1998). In each clinical situation, the choice of the surgical technique to be employed depends on various factors, such as the size of the lesion, site of injury and activity and age of the patient (Valderrabano et al., 2013; Vasiliadis and Wasiak, 2010). Techniques such as abrasion and drilling are considered by the surgeon only in a few particular cases (Vasiliadis and Wasiak, 2010). Microfracture is a widely used technique and it is considered the treatment of choice for the healing of small OCDs, i.e. not exceeding $1.5 \mathrm{~cm}^{2}$ (Valderrabano et al., 2013). In this particular condition, the use of mosaicplasty, namely osteochondral autotransplantation, is also supported (Valderrabano et al., 2009).

For larger lesions and/or patients with moderate to high activity, new approaches using techniques inspired by regenerative medicine principles have been applied, showing promising results (Giannini et al., 2008; Giza et al., 2010; Gobbi et al., 2006). One of the most encouraging treatments comprises cell-based therapies, such as autologous chondrocyte implantation (ACI). Based on tissue engineering (TE) techniques, implantation of matrixassociated cells has also been attempted, using stem cells (MASI) or chondrocytes (MACI), either at the ankle or knee (Schneider and Karaikudi, 2009; Zengerink et al., 2010). In fact, TE of the ankle has been attracting a great deal of interest (Correia et al., 2014). Although promising, the MASI approach still requires better control of stem cells chondrogenic differentiation in vivo as compared to MACI. Therefore, the latter has been more widely applied. The main advantage of MASI over MACI is that it may require a one-step (Giannini et al., 2009) instead of the two-step procedure, thus eliminating the cost and timeconsuming process of culture expansion.

For procedures using chondrocytes, the cells are commonly harvested from the healthy cartilage (Candrian et al., 2010), usually from the ipsilateral knee or from the anterior part of the talus (Baums et al., 2006), and are further expanded in vitro (Vasiliadis and Wasiak, 2010). In a second stage, chondrocytes are implanted in the defect directly or after 'seeding' into a three-dimensional (3D) porous matrix or scaffold (Valderrabano et al., 2013; Vannini et al., 2013; Vasiliadis and Wasiak, 2010). However, this technique is frequently associated with pain at the donor site (Reddy et al., 2007; Valderrabano et al., 2009) and is detrimental to the function of the ankle joint (Candrian et al., 2010). Up to 50\% incidence of knee pain has been described post-transplant of donor knee-ankle (Reddy et al., 2007; Valderrabano et al., 2009). A convenient approach would be the utilization of chondrocytes isolated from OCDs that are usually harvested during surgery. In fact, it was reported that these chondrocytes can be used in ACI or MACI techniques with good clinical results (Giannini et al., 2005). However, the chondrogenic capacity of chondrocytes isolated from OCDs remains controversial, since it has been reported that the formed tissue contains an inferior amount of glycosaminoglycan and type II collagen and a superior amount of type I collagen (Kuroki et al., 2002), as well as a reduced cartilage-forming capacity (Candrian et al., 2010). Therefore, this approach does not seem to be the most adequate strategy when envisioning the treatment of ankle OCDs, at least for now.

The posterior process of the talus, also known as Stieda's process, can be associated with the syndrome of posterior impingement of the talus, especially in athletes and ballet dancers (Bizarro, 1921; Russell et al., 2010). Its clinical management requires arthroscopic surgery, in which Stieda's process is removed (Park et al., 2013). Also described in the literature is the existence of an accessory bone, the os trigonum, which is associated with the posterior process of the talus and has no defined function. Nevertheless, it has been frequently associated with chronic complaints related to plantar flexion mechanisms (Bizarro, 1921). In the symptomatic posterior ankle impingement syndrome, the removal of these structures has been described and proposed for pain management (Park et al., 2013; Russell et al., 2010). The orthopaedic community assumes that the arthroscopic excision of these structures is not associated with significant morbidity (Chao, 2004). Due to the minimally invasive surgery generally used during its excision (Tey et al., 2007; van Dijk et al., 2000), this tissue could be seen as a potential valuable source of cells for ACI or MACI procedures.

To our knowledge, there are no reports on the biological characterization of the posterior talus process or the os trigonum, so basic studies are greatly needed. This work aims to characterize the posterior process of the talus and os trigonum tissues in order to achieve a new source of viable cells for use in tissueengineering approaches and treatment of OCDs of the ankle. The biological tissue from six patients who underwent excision of the osteochondral fragments was characterized in terms of tissue architecture, composition of extracellular matrix and cellularity by micro-computed tomography (micro-CT) analysis, immunohistochemistry and histomorphometric analysis, respectively. Moreover, cells isolated from the extracted tissue were evaluated in vitro, for up to 21 days of culture, regarding their proliferation ability, viability and occurrence of cell apoptosis by DNA quantification, live/dead and MTS assay and TUNEL reaction method, respectively. Cells were also characterized regarding the expression of specific surface markers by flow cytometry, in order to determine cells phenotype. 


\section{Materials and methods}

\subsection{Biological samples}

Human tissues were obtained through cooperation protocols established with Centro Hospitalar Póvoa do Varzim and do Dragão. The study was approved by the Ethical Committee of Centro Hospitalar Póvoa do Varzim and the University of Minho. The ankle tissues comprising the posterior process of the talus (SP), os trigonum (OT) and normal ankle articular cartilage (AJ) (see supporting information, Figure S1) were obtained from different age and sex donors by means of surgical technique. It was guaranteed that patients were enrolled for surgery (excision of osteochondral fragment) regardless of study participation. Patients received information about the procedure and were selected to participate in the study after obtaining their informed consent in written form. There was no interference in the ordinary course of treatment. Biological samples $(n=6)$ were then selected for tissue $(n=3)$ and cellular $(n=3)$ characterization. The samples were classified as OT or SP, as indicated by the surgeon. One of the samples was obtained from the AJ, and it was also processed for biological characterization according to the established protocols for OT and SP. The tissue was maintained in a phosphate-buffered saline (PBS; Sigma) solution containing $1 \% \mathrm{~V} / \mathrm{v}$ antibioticantimycotic mixture (AB; Gibco) and processed within $24 \mathrm{~h}$ for cell isolation. For studying tissue architecture and histological studies, the extracted tissues were rinsed with PBS and fixed in 10\% v/v formalin (Bio Optica) for at least 1 day.

\subsection{Tissue characterization}

\subsubsection{Micro-computed tomography (micro-CT) analysis}

The microstructure of the extracted tissues $(n=3)$ was qualitatively and quantitatively evaluated by micro-CT analysis. The structure of the sample was acquired by X-ray and then reconstructed and analysed. Hard/soft tissues (approximately corresponding to bone and cartilage, respectively) morphometric parameters, such as porosity, mean pore size, trabecular thickness and hard/soft tissues profiles, were determined. A 3D reconstruction was also performed in order to characterize the tissue. Data acquisition was performed in a SkyScan 1072 scanner with a pixel size of $16 \mu \mathrm{m}$ and an integration time of $1280 \mathrm{~ms}$. The X-ray source was set at $82 \mathrm{kV}$ and $122 \mu \mathrm{A}$. Approximately 600 projections were acquired over a rotation range of $180^{\circ}$, with a rotation step of $0.45^{\circ}$. Datasets were reconstructed using standardized cone-beam reconstruction software (NRecon v. 1.6.6.0, SkyScan). The output format for each sample was bitmap images. The set of images was orientated with DataViewer (v. 1.4.4, SkyScan) to obtain all samples in the same axis. A representative dataset of the slices was segmented into binary images with a dynamic threshold of 40-120 for soft tissue and 120-255 for hard tissue analysis (grey values). Afterwards, the binary images were used for morphometric analysis (CT Analyser, v. 1.12.0.0, SkyScan) and to obtain the 3D models (CT Vox, v. 2.3.0 r810, SkyScan).

\subsubsection{Histological and immunohistochemical characterization}

Specimens $(n=3)$ were decalcified with $10 \% \mathrm{v} / \mathrm{v}$ formic acid (Panreac) for 2 weeks. After this step, the samples were processed in a spin tissue processor (STP120-2, Thermo Scientific) overnight, which included immersion in baths of formalin, ascending series of ethanols (Panreac), xylene (VWR International) and paraffin impregnation (Thermo Scientific). The specimens were then embedded in paraffin and sectioned into $3 \mu \mathrm{m}$ slices using a microtome (HM 355 S, MICROM International $\mathrm{GmbH}$ ). Three different stainings were performed: haematoxylin and eosin (H\&E), Masson's trichrome (MT) and toluidine blue (TB). H\&E was used to visualize the tissue structure and architecture, as well as cellularity. MT and TB were performed to detect articular cartilage extracellular matrix components, such as collagen and glycosaminoglycans, according to previously described methods (van Dijk et al., 2000). Slides were then imaged under transmission microscopy through an Axio Imager.Z1m light microscope (Zeiss) with an attached digital camera, AxioCam MRc5 (Zeiss), connected to ZEN blue image processing software (Zeiss). For immunohistochemistry, sections were blocked with sodium citrate buffer (10 mM sodium citrate in $0.05 \%$ Tween 20; Aldrich) and stained with the respective two primary antibodies and fluorescentlabelled secondary antibody (see supporting information, Table S1). Counterstaining of cell nuclei was further performed with 4,6-diamidino-2-phenyindole, dilactate (DAPI, Sigma). Sections were imaged under fluorescence microscopy using an AxioCam MRc5 digital camera (Zeiss) connected to ZEN blue image processing software (Zeiss) attached to the Axio Imager.Z1m light microscope (Zeiss).

\subsubsection{Histomorphometric analysis}

The images obtained from the slides stained with H\&E were used to perform a histomorphometric analysis to determine the chondrocyte density on cartilage segments. The WCIF ImageJ software program (US National Institutes of Health) was used for cell counting. After subtracting the background, a 12-radius threshold was used to reduce background noise and automatic cell counting (nuclei) was performed. A total of 50 photomicrographs $\left(700 \times 500 \mu \mathrm{m}^{2}\right) /$ specimen were analysed $(n=3)$. 


\subsection{Cells biological characterization}

\subsubsection{Cell isolation and expansion}

Chondrocytes populations $(n=3)$ were isolated following an enzymatic digestion-based method with type II collagenase (Sigma) from ankle tissues. The extracted tissue was placed in PBS solution and washed several times with PBS containing $1 \% \mathrm{v} / \mathrm{v} \mathrm{AB}$ mixture, until total removal of blood or other bodily contaminants was achieved. Cartilage tissue was then separated from bone and, after being washed several times with PBS solution containing 1\% $\mathrm{v} / \mathrm{v} A B$ mixture, the cartilage portion was cut into small pieces. Tissue digestion was performed by incubation at $37^{\circ} \mathrm{C}$ in a humidified atmosphere of $5 \% \mathrm{CO}_{2}$ for $1-2 \mathrm{~h}$ in $10-20 \mathrm{ml}$ of a mixture composed of Dulbecco's modified Eagle's medium F12 (DMEM F12; Gibco), supplemented with $10 \%$ fetal bovine serum (FBS; Gibco), 1\% AB mixture and type II collagenase (1:1). The digested tissue was filtered and the cell suspension centrifuged at $1220 \mathrm{rpm}$ for $5 \mathrm{~min}$. The isolated cell populations were expanded in DMEM F12 supplemented with 10\% FBS and 1\% AB mixture until confluence was reached. Chondrocytes were expanded until passage 3 and detached from the T-75 culture flask using TrypLE Express (Gibco). Polystyrene tissue culture was performed in a 24-well plate, using cells at passage 4 with a cell density of 10000 cells/well. Each sample was evaluated after specific periods of culture (time points, TPs), i.e. 1, 3, 7, 14 and 21 days, in triplicate for each tissue type, and three wells without cells were used as control. Two independent sets of experiments were performed.

\subsubsection{Cellular viability and proliferation}

Cell metabolic activity was assessed by performing MTS [3(4,5-dimethylthiazol-2-yl)-5(3-carboxymethoxyphenyl)2(4-sulphofenyl)-2H-tetrazolium] assay (Cell Titer $96^{\circledR}$ Aqueous Solution Cell Proliferation Assay, Promega), according to the ISO/EN 10993 Part 5 guidelines (ISO/ EN10993-5, 2009). At each TP (i.e. 1, 3, 7, 14 and 21 days), cultured cells were washed with PBS solution and incubated in MTS reagent at $37^{\circ} \mathrm{C}$ in a $5 \% \mathrm{CO}_{2}$ atmosphere for $3 \mathrm{~h}$ in the dark. After incubation, $100 \mu \mathrm{l}$ of each well (in triplicate) were transferred into a 96-well plate for optical density (OD) measurement at $490 \mathrm{~nm}$ in a microplate reader (Molecular Devices). The percentage of cell viability was calculated after normalization with the mean OD value obtained for the negative control. OD is directly proportional to the cellular activity, being a measure of mitochondrial activity.

Detection and quantification of DNA was performed by using the Quant-i ${ }^{\mathrm{TM}}$ PicoGreen ${ }^{\circledR}$ dsDNA Reagent (Invitrogen). After washing the cells with PBS, $1 \mathrm{ml}$ ultra-pure water was added to each well. The cells were then mechanically detached from the well and the suspension was transferred into Eppendorf tubes. After incubation in a water bath at $37^{\circ} \mathrm{C}$ for $1 \mathrm{~h}$, the cell suspension was frozen at $-80^{\circ} \mathrm{C}$ for at least $1 \mathrm{~h}$. Afterwards, the mixture was defrosted in an ultrasonic water bath (DT100H Sonorex Digitec, Bandelin) for $15 \mathrm{~min}$ and transferred in triplicate into a 96-well plate. PicoGreen ${ }^{\circledR}$ solution was added afterwards in a 1:1 ratio. Fluorescence was measured in a microplate reader at ex/em $\sim 480 / 520 \mathrm{~nm}$. $\lambda$ DNA (Invitrogen) was used to prepare the standard curve.

Cell viability was evaluated by live/dead assay after culturing the cells in $13 \mathrm{~mm}$ tissue culture coverslips. Live cells (stained with calcein AM 2:1000, Gibco; ex/em $\sim 495 / 515 \mathrm{~nm}$ ) and dead cells (indicated by propidium iodide 1:1000, Gibco; ex/em $\sim 495 / 635 \mathrm{~nm}$ ) were imaged through a Zeiss Axio Imager.Z1m fluorescence microscope.

\subsubsection{Cellular apoptosis}

The in situ Cell Death Detection Kit (Roche) was used to detect apoptotic cells, using the terminal deoxynucleotidyl transferase dUTP nick end labelling (TUNEL) reaction. Briefly, cells cultured in $13 \mathrm{~mm}$ tissue culture coverslips were washed in PBS and fixed in $10 \% \mathrm{v} / \mathrm{v}$ formalin for $1 \mathrm{~h}$ at room temperature. Afterwards, the cells were incubated in a permeabilization solution $(0.1 \%$ Triton $\mathrm{X}$ in $0.1 \%$ in sodium citrate, Sigma) for $2 \mathrm{~min}$ on ice. Negative (without terminal transferase) and positive (with DNase I recombinant $20 \mathrm{U} / \mathrm{ml}$; Amresco) controls in $50 \mathrm{~mm}$ Tris(hydroxymethyl) aminomethane (Sigma), $\mathrm{pH} 7.5$, and $1 \mathrm{mg} / \mathrm{ml}$ bovine serum albumin (Sigma) were also prepared. For each sample, $50 \mu 1$ TUNEL reaction mixture was added and incubated at $37^{\circ} \mathrm{C}, 5 \% \mathrm{CO}_{2}$ for $1 \mathrm{~h}$ in the dark, as well as on the positive and negative controls. Counterstaining with fluorescein phalloidin (Sigma) and DAPI was also performed. Apoptotic cells were imaged using a Zeiss Axio Imager.Z1m fluorescence microscope (ex/em $\sim 495 / 515 \mathrm{~nm}$ ) and phalloidin (ex/em $\sim 540 / 565 \mathrm{~nm}$ ) and DAPI (ex/em 358/461 nm) were used to double-check the location of cells and their nuclei.

\subsubsection{Matrix production evaluation}

Evaluation of matrix production was performed by toluidine blue $\mathrm{O}$ (Betalab) staining at $1 \% \mathrm{w} / \mathrm{v}$ in cells grown in $13 \mathrm{~mm}$ tissue culture coverslips for each period of culture. Images were obtained under transmission microscopy through an Axio Imager.Z1m light microscope.

\subsubsection{Phenotype characterization}

Fluorescence-activated cell sorting (FACS) analysis was performed to examine surface marker expression on chondrocytes at passages 2 and 3 following isolation from cartilage samples $(n=3)$. Cells were tested using anti-human monoclonal fluorescein isothiocyanate (FITC)-conjugated mouse CD105 (AbD Serotec), CD90 and CD45 (BD Biosciences) antibodies; phycoerythrin (PE) conjugated mouse CD73, CD44 and CD34 (BD Biosciences) antibodies; and allophycocyanin (APC) 
conjugated rat CD49f (eBioscience) and mouse CD90 (BD Biosciences) antibodies. All antibodies were used at concentrations recommended by the manufacturers for FACS analysis. Briefly, cells frozen at different passages were thawed, collected in culture media and counted using the trypan blue exclusion method. A cell suspension containing a total of $6 \times 10^{5}$ cells was centrifuged for $5 \mathrm{~min}$ at $300 \times \mathrm{g}$ and the pellet was resuspended in $500 \mu \mathrm{l}$ PBS containing $2 \% \mathrm{w} / \mathrm{v}$ BSA (Sigma). For direct staining, $1.5 \times 10^{5}$ cells/tube were incubated for $15 \mathrm{~min}$ with fluorochrome-conjugated antibodies at room temperature in the dark. After incubation, the cells were washed with PBS/BSA solution, centrifuged for $5 \mathrm{~min}$ at $1500 \mathrm{rpm}$, resuspended in PBS and analysed with counting of a minimum of 40000 events. Calibrite beads three-colour kit (BD CaliBRITE ${ }^{\mathrm{TM}}$ beads, BD Biosciences) was used to adjust the equipment instrument settings before running the samples. The data were acquired using a BD FACSCanto ${ }^{\mathrm{TM}}$ (BD Biosciences) flow cytometer. Labelling experiments were repeated three times.

\subsection{Statistical analysis}

Statistical analysis was performed using commercial SPSS software (IBM SPSS Statistics v. 21.0, IBM). ANOVA Bonferroni was used to assess significant differences in the mean values of cell counting on each sample. Paired samples $t$-tests were used to evaluate significant differences in the mean values of absorbance/DNA concentration between time points. Complementarily, Cohen's $d$-test was used. Significant differences between samples were assessed with ANOVA Bonferroni. In all cases, the level of significance used was set at $p<0.05$ for a $95 \%$ confidence interval.

\section{Results}

\subsection{Tissue characterization}

Two samples of the posterior process of the talus (SP) and one of the os trigonum (OT) explants were characterized by micro-computed tomography (micro-CT) analysis and histology/immunohistochemistry, and were designated by SP1, SP2 and OT, respectively. The mean age of the patients was $38 \pm 12$ years.

The structure of the samples was acquired by X-ray and then reconstructed and analysed. Hard/soft tissues (approximately corresponding to bone and cartilage, respectively) morphometric parameters, such as porosity, mean pore size, trabecular thickness and hard/soft tissues profiles, were determined. A 3D reconstruction was also performed in order to characterize the tissue. The data obtained in micro-CT analysis (Table 1) indicated values of porosities for OT, SP1 and SP2 of 90\%, $93 \%$ and $98 \%$ in hard tissue and $68 \%, 77 \%$ and $76 \%$ in soft tissue, respectively. Mean trabecular thickness in hard tissue was 242, 214 and $162 \mu \mathrm{m}$ for OT, SP1 and
Table 1. Structural properties of hard and soft tissues in three ankle explants

\begin{tabular}{lrrr}
\hline & OT & SP1 & SP2 \\
\hline Hard tissue/bone & & & \\
Mean porosity (\%) & 90 & 93 & 98 \\
Mean trabecular thickness $(\mu \mathrm{m})$ & 242 & 214 & 162 \\
Mean pore size $(\mu \mathrm{m})$ & 2247 & 3970 & 2377 \\
Interconnectivity $(\%)$ & 92 & 90 & 99 \\
Soft tissue/cartilage & & & \\
Mean porosity (\%) & 68 & 77 & 76 \\
Mean trabecular thickness $(\mu \mathrm{m})$ & 278 & 177 & 251 \\
Mean pore size $(\mu \mathrm{m})$ & 1194 & 2906 & 2203 \\
Interconnectivity $(\%)$ & 79 & 80 & 84 \\
\hline
\end{tabular}

OT, os trigonum; SP1 and SP2, Stieda's processes.

SP2, respectively. In soft tissue, the values of mean trabecular thickness were 278, 177 and $251 \mu \mathrm{m}$ for OT, SP1 and SP2, respectively. Similarly to the parameter of mean porosity, the pore size was larger in hard tissue in all samples. For all specimens, the cartilage tissue represented at least $20 \%$ on average. The OT has a higher percentage of cartilaginous tissue, i.e. $32 \%$ as compared to SP1 and SP2. Interconnectivity was higher in hard tissue as compared to soft tissue.

Figure 1 shows the micro-CT morphometric analysis of the human ankle explants. All the explants reached approximately a $50 \%$ content in soft tissue (Figure 1A). The distribution of hard tissue trabecular thickness (Figure 1B) showed that about $36 \%$ of the trabecular diameters were in the range $181.3-280.2 \mu \mathrm{m}$. From the data obtained for soft tissue thickness (Figure 1B), it was possible to observe that around $22 \%$ of the diameters were in the range 155.4-181.3 $\mu \mathrm{m}$, which indicated that soft tissue presents inferior trabecular diameters as compared to hard tissue. In terms of hard tissue pore size, the profile showed that the majority of the pores had diameters $>80-110 \mu \mathrm{m}$. Compared with hard tissue pore size, a higher variation in pore size values was found in soft tissue for all explants. Moreover, soft tissue appeared to have two types of pores: a smaller range $<10 \mu \mathrm{m}$ and a larger range of pores that started at approximately $50 \mu \mathrm{m}$ and reached $>100 \mu \mathrm{m}$. Figure 2 shows 2D (X-ray) and 3D reconstructed images of the human ankle OT and SP explants. It is possible to observe two distinct regions, corresponding to cartilage (external part) and bone (core region) tissues.

Figure 3 shows optical microscopy images with different staining for each specimen. H\&E staining was used to visualize the tissue structure and architecture, as well as cellularity; MT and TB staining revealed the presence of negatively charged glycosamicoglycans and proteoglycans within the cartilage. The deposition of type II collagen was also detected on cartilage tissue by immunohistochemical analysis. Although the presence of type I collagen was also evaluated (see supporting information, Figure S2), it was not possible to be detected, due to the natural fluorescence of the specimens.

The quantification of cells in SP and OT was performed using WCIF ImageJ software, in order to evaluate chondrocyte density within the cartilage in this kind of tissue. 

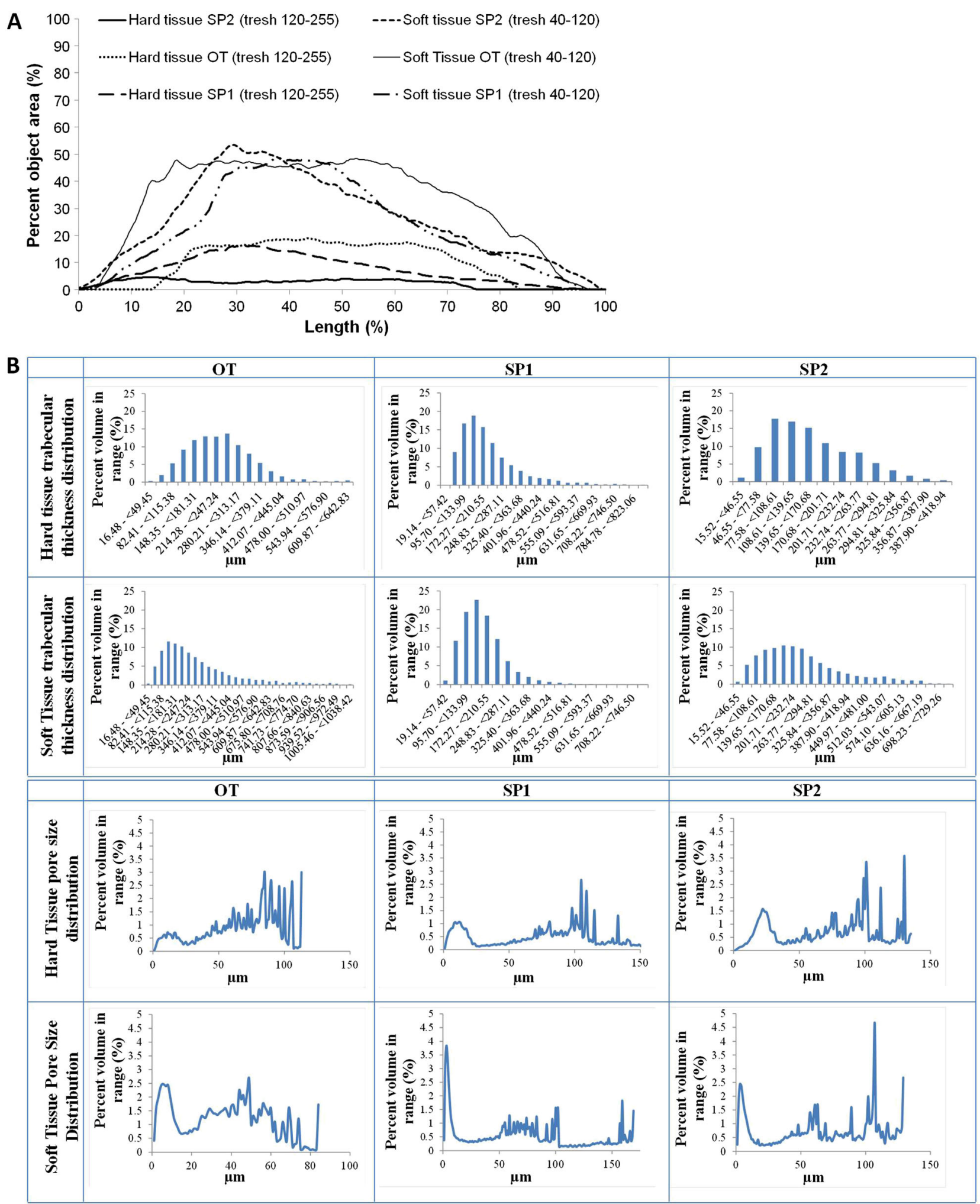

Figure 1. Micro-CT morphometric analysis of human ankle explants: (A) percentages of soft and hard tissue; (B) trabeculae and pore size distributions in the hard and soft tissue of the explants; OT, os trigonum; SP, Stieda's process

Cell density observed in cartilage had a mean value of 350 \pm 101 cells $/ \mathrm{mm}^{2}$ for OT, $204 \pm 65$ cells $/ \mathrm{mm}^{2}$ for SP1 and $342 \pm 76$ cells $/ \mathrm{mm}^{2}$ for SP2 (see supporting information, Figure S3). Significant differences were observed in average cell counts $/ \mathrm{mm}^{2}$ between SP1 and SP2, as well as between SP1 and OT $(p<0.001)$. No significant differences were observed between SP2 and OT $(p>0.5)$.

\subsection{Cells biological characterization}

The ages of tissue donors for cell isolations were 35 (SP), 19 (OT) and 23 (AJ) years, with an average age of $26 \pm 7$ years. The enzymatic method used for chondrocytes isolation from the ankle tissues proved to be reliable, as it consistently permitted to obtain 
Ankle explant 2D (X-ray)

OT

SP1

SP2

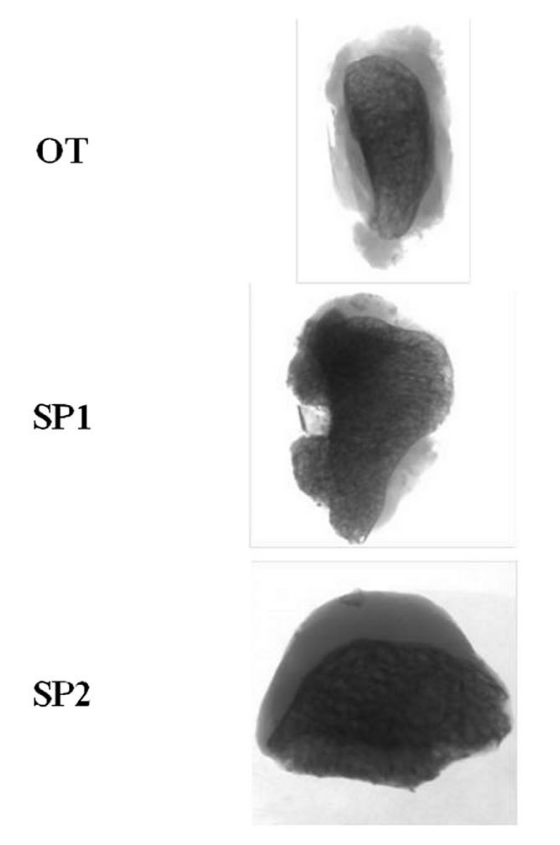

3D (Hard tissue)
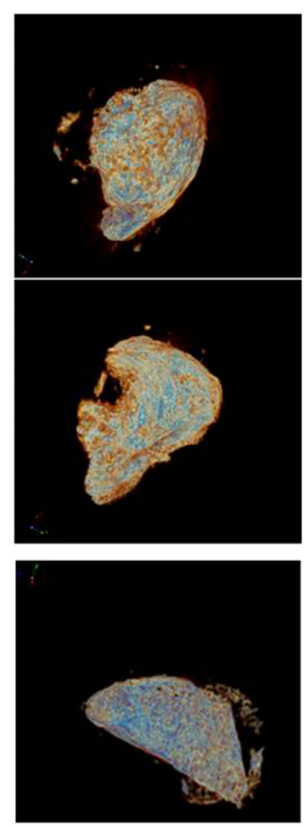

3D

(Soft+Hard tissues)
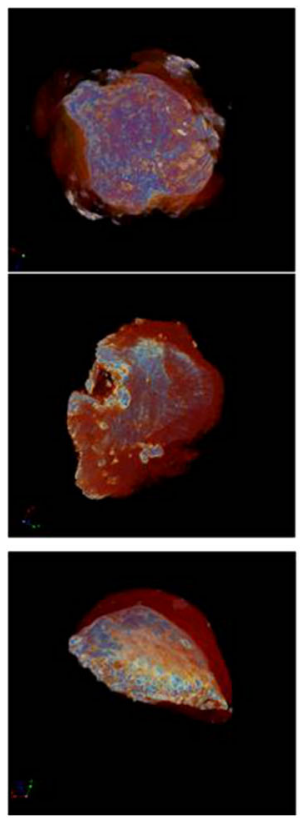

Figure 2. 2D and 3D images of human ankle explants obtained by micro-CT analysis; OT, os trigonum; SP, Stieda's process

viable cells. Isolated cells presented a round morphology typical of chondrocytes. At passage 4, cells cultured for different time periods (i.e. 1, 3, 7, 14 and 21 days) exhibited a similar elongated and spindleshaped morphology characteristic of dedifferentiated chondrocytes.

The metabolic activity of the isolated chondrocytes was quantified by reduction of MTS reagent at each culture time for the three types of tissue (Figure 4A). The results showed that cells remained metabolically active at each time point (TP) tested. For each sample, differences between two consecutive TPs were significant $(p<0.05)$, except for TP_14d-TP_21d ( $d=$ days) on the AJ sample, where no significant differences were observed. Within each TP, significant differences were observed between each type of cell in most cases $(p<0.05)$. Cell proliferation was evaluated through quantification of DNA of the cultured cells at each period of culture (Figure 4B). The DNA concentration increased significantly along the culture period, except on TP_21d of the OT sample, where a significant decrease was observed $(p<0.001)$. Significant differences were observed between the three samples tested for all the periods of culture, except on TP_1d between SP and AJ and TP_21d between SP and OT, where no significant differences were observed $(p>0.05)$.

Live/dead imaging of the different groups corroborated the viability (MTS) and proliferation (DNA quantification) studies, as viable cells (green fluorescence) were observed by fluorescence microscopy (Figure 5). The number of viable cells increased from day 1 until day 21 of culture.

Cellular apoptosis was investigated by means of performing TUNEL assay (Figure 6). This study also corroborated the live/dead findings, as no apoptosis was visualized, i.e. no green fluorescence (upper right images) was observed for the great majority of the cells at each culture time.

Matrix production and deposition (i.e. proteoglycans and collagen) was also evaluated with TB staining in each period of culture for each specimen. No relevant matrix deposition was observed from day 1 until day 21 in all types of cells (Figure 7).

For performing phenotypic characterization, ankle chondrocytes were isolated from tissue harvested from three independent donors (mean age $26 \pm 7$ years). Cells were cultured until reaching confluence and trypsinized at passages 2 and 3 . Subsequently the cell population was analysed for surface antigens by flow cytometry. Flow-cytometer calibration was based on autofluorescent expression of FITC, APC and PE by isolated chondrocytes. The cells do not express their own fluorescence at any of the channels used, as confirmed in Figure 8A for FITC and representative for the APC and PE channels. Fluorescent distribution of the different chondrogenic and MSC surface markers was evaluated at passages 2 and 3 of cell populations. Figure 8B represents the positive fluorescent distribution of FITC and Figure 8C the absence of fluorescence expression. The surface screening showed that cells maintained expression of the chondrogenic surface markers CD105, CD90, CD73, CD49f and CD44, while showing a very low expression of MSC markers CD45 and CD34, in the living cells between passages 2 and 3, confirming the maintenance of the chondrogenic phenotype of the cells isolated from ankle tissues during culture (Figure 8D). 

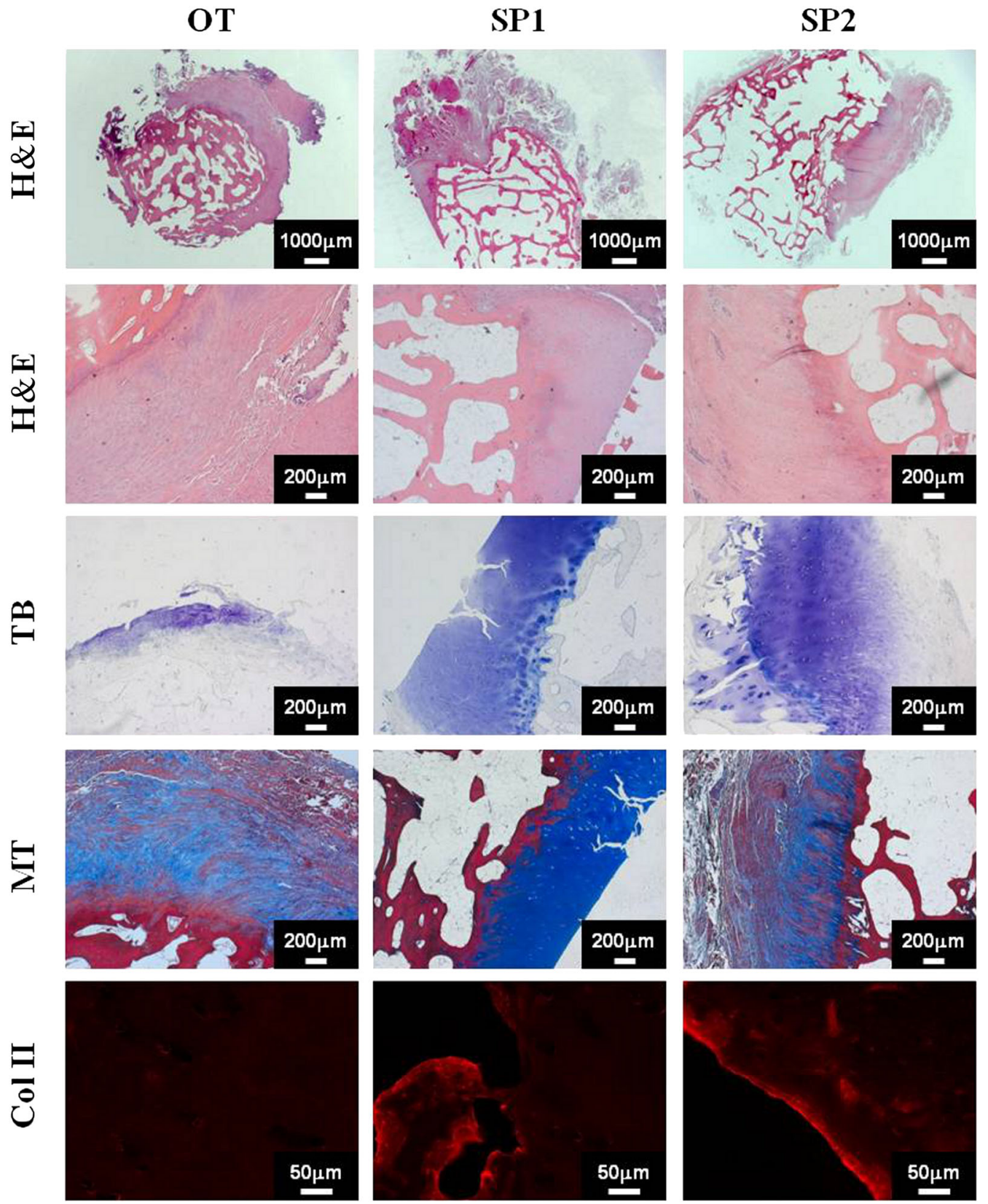

Figure 3. Microscopy images of the histological sections of cartilage: rows 1-4, histological stainings of cartilage extracellular matrix; row 5, immunohistochemical localization of type II collagen; H\&E, haematoxylin and eosin; TB, toluidine blue; MT, Masson's trichrome; Col II, type II collagen; OT, os trigonum; SP, Stieda's process

\section{Discussion}

In this study, the posterior process of the talus (SP), os trigonum (OT) and normal articular ankle cartilage (AJ) tissues and isolated cells were characterized. Cells isolated from SP, OT and AJ were compared in terms of viability and proliferation in $2 \mathrm{D}$ cultures, as well as in their post-expansion extracellular matrix-forming capacity.

As previously shown in Figure S1A (see supporting information), the SP and OT structures are closely related to the talus. Characteristically, the OT is not fused to the talus, whereas the SP is described as a prominent elongated posterolateral process of the talus (Rathur et al., 2009). From micro-CT analysis, hard tissue and soft tissue can be associated with bone and cartilage fractions, respectively. In the three explants, cartilage reached approximately $50 \%$ of the total tissue content. OT showed a higher content in cartilage tissue as compared to SP. Our results from micro-CT morphometric analysis showed that there is soft tissue completely surrounding the hard tissue. Nevertheless, all the explants presented high amounts of cartilage, which supports the hypothesis in our study. Our findings were corroborated by the histological studies, where staining by MT and TB denoted the presence of cartilage-like matrix. In SP1, the blue staining was more intense than in SP2 and OT. It was also possible to observe the appearance of typical hyaline cartilage, with isolated and rounded chondrocytes surrounded by the cartilaginous extracellular matrix 

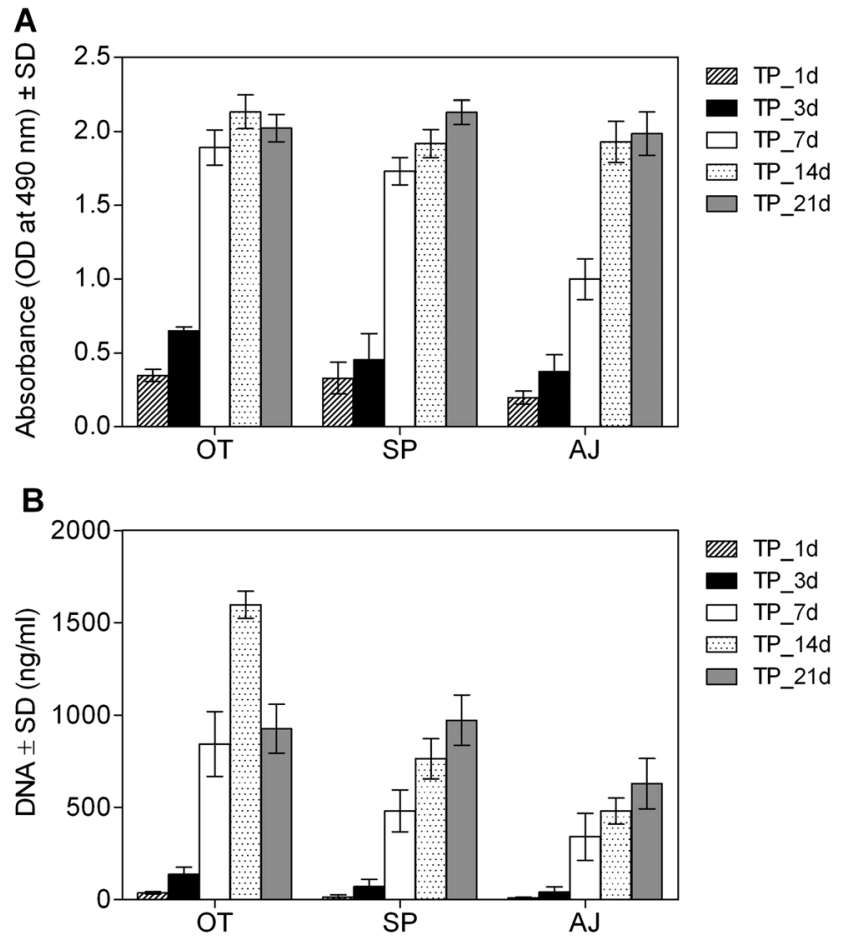

Figure 4. Evaluation of biological performance of cells isolated from ankle tissues and cultured for 1, 3, 7, 14 and 21 days (d). (A) Evaluation of cell viability: statistically significant differences were observed between time points for each sample $(p<0.05$, d $>0.8$ ). (B) Assessment of DNA content: statistically significant differences were observed between time points for each sample ( $p<0.001, \mathrm{~d}>0.8$ ); OT, os trigonum; SP, Stieda's process; AJ, ankle joint; TP, time point

(Junqueira et al., 2007). On the other hand, OT and SP2 were showed to possess less hyaline cartilage content. Fusiform chondrocytes interspersed with collagen fibres were observed in OT and SP2, which is typical of fibrocartilage. This type of cartilage presents mainly type I collagen fibres instead of the characteristic type II collagen of hyaline cartilage. Nevertheless, type II collagen was found in all characterized explants, as demonstrated by immunohistochemical analysis. While no considerations could be made based on these results regarding type I collagen deposition, it is not possible to conclude that SP2 and OT present higher amounts of fibrocartilaginous tissue. SP and OT did not possess higher amounts of hyaline cartilage, which was expected, since these tissues are not anatomically confined to an articular area, where hyaline cartilage is indicative of healthy articular cartilage. Despite the type of cartilage present in OT or SP, the presence of viable chondrocytes supports the study hypothesis. Several other studies have reported the ability of chondrocytes to produce hyaline matrix, i.e. deposition of glycosaminoglycans and type II collagen, under chondrogenic medium and in the presence of growth factors (Giannini et al., 2005; Tay et al., 2004). It is known that during monolayer expansion, chondrocytes undergo a process of dedifferentiation, acquiring a fibroblast-like phenotype and exhibiting a certain degree of plasticity, being able to differentiate towards the chondrogenic phenotype in response to specific microenvironmental factors (Barbero et al., 2006; Benya and Shaffer, 1982).

Once again, in histomorphometric analysis, the average number of cells $/ \mathrm{mm}^{2}$ in SP1 was significantly different $(p<0.001)$ as compared to SP2 and OT. This corroborates the assumption of a larger amount of hyaline cartilage in SP1 explants, contrarily to SP2 and OT, where no significant differences $(p>0.05)$ were observed. Due to the typical wide matrix deposition that occurs in hyaline cartilage, the cells are more widely spaced, which determines a lower cell number $/ \mathrm{mm}^{2}$ tissue. Despite the observed differences in cell numbers for the two tissues, a significant number of cells/area of tissue was observed and can be isolated, supporting the idea that chondrocytes can be obtained in enough number for possible use in regenerative medicine approaches.

In this study, it was also demonstrated that it is feasible to isolate viable chondrocytes from the SP and OT. The in vitro studies demonstrated that isolated cells proliferated and remained metabolically active for up to 21 days of culture. In fact, a major increase in viability and proliferation was observed from day 3 to day 7. Moreover, between day 7 and day 14, a significant increase $(p<0.001)$ in viability and proliferation was also observed. As expected, the proliferation was not so expressive at day 21 , as a consequence of confluence-induced cell death. A decrease in DNA content was also observed in OT isolated cells at day 21, possibly due to their high proliferation ability, as depicted by the high DNA content observed at day 14. These results thus showed that isolated ankle cells can grow on 2D cell culture and remain viable for at least 21 days. Furthermore, not only the cells were viable, they were also non apoptotic, as demonstrated by the TUNEL assay. The differences observed between SP, OT and AJ in terms of viability and proliferation within each time period, although significant $(p<0.05)$, do not represent a limitation for further use of chondrocytes isolated from these tissues. In fact, DNA content in AJ at day 21 is significantly inferior as compared to SP and OT. Several studies have reported the use of AJ chondrocytes for autologous chondrocyte implantation (ACI) and matrix associated-cells implantation (MACI) strategies, with promising clinical results (Aurich et al., 2011; Correia et al., 2014; Giannini et al., 2008; Giza et al., 2010). Aurich et al. (2011) and Giannini et al. (2008) reported a significant improvement in all clinical scores after MACI and ACI procedures, respectively, using chondrocytes isolated from AJ. In this study, isolated OT and SP chondrocytes presented better results in terms of viability and proliferation, which indicates that chondrocytes isolated from OT and SP could also be potentially used for MACI and ACI procedures.

In a study comparing chondrocytes obtained from naturally occurring ankle osteochondral defects (OCDs) and AJ cartilage tissue, OCD chondrocytes were less capable of appropriate extracellular matrix production than chondrocytes from unaffected ankle joints (Schachter et al., 2005). Also, a recent study has shown that chondrocytes obtained from the injured zone in the 

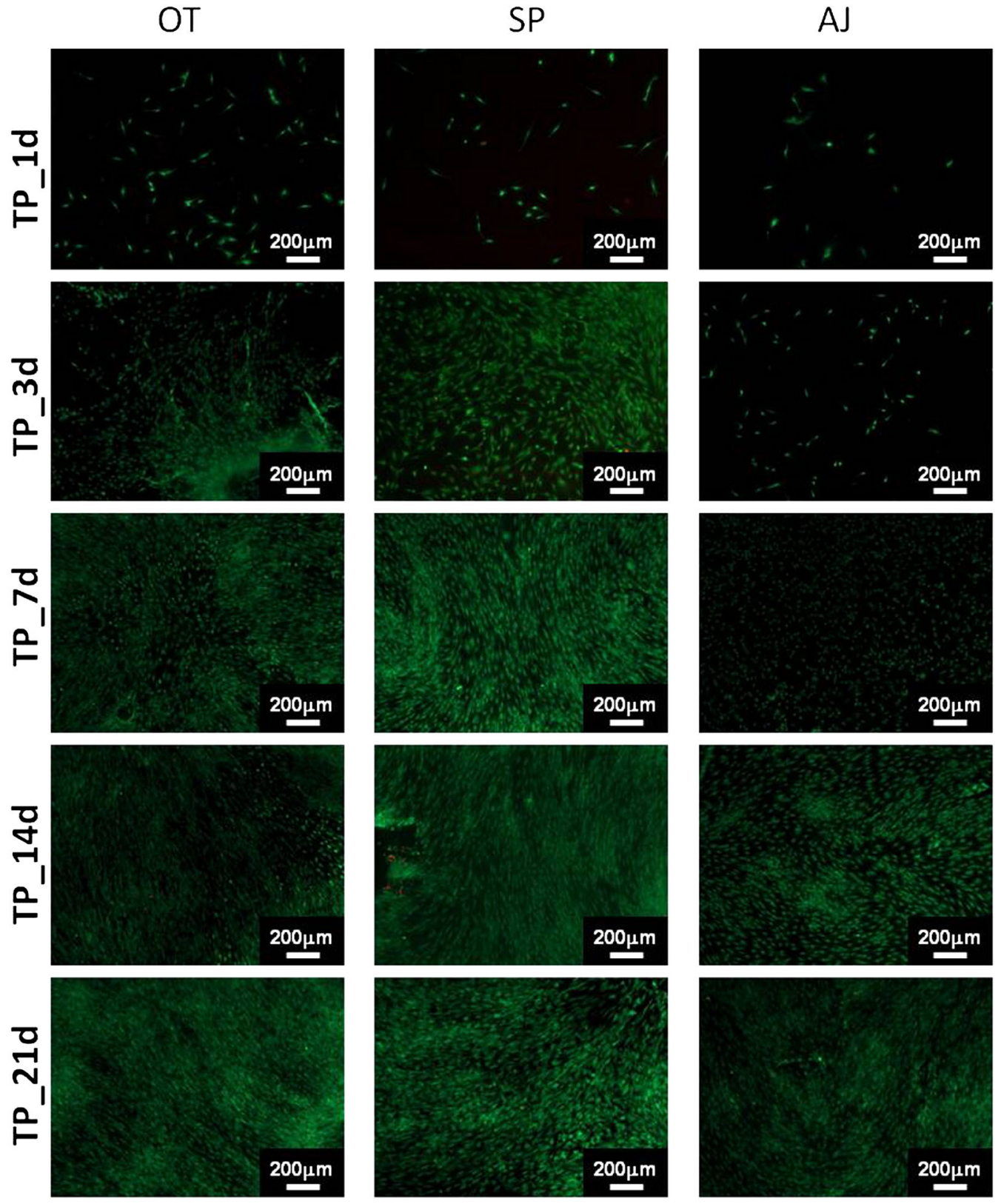

Figure 5. Live/dead imaging of cells isolated from ankle tissues and cultured in vitro for 1-21 days (d); OT, os trigonum; SP, Stieda's process; AJ, ankle joint; TP, time point

ankle have inferior regenerative capacities as compared to those from normal tissue, suggesting some reservations concerning their widespread use in the therapeutic field (Candrian et al., 2010). The use of healthy cartilage therefore seems to be more reliable to obtain good clinical results.

In the present in vitro studies, standard culture medium was used and no chondrogenic supplements were added. Thus, no relevant matrix production or deposition was observed until day 21 , as expected.

Fluorescence-activated cell sorting (FACS) was performed to determine the chondrogenic capacity of the isolated cells, using surface chondrocyte membrane markers (i.e. CD105, CD90, CD73, CD49f and CD44). Because chondrocytes are the main entities for extracellular matrix deposition in cartilage tissue, an alteration in the cellular phenotype has an enormous significance on the type of matrix (Garvican et al., 2008). In the present study, flow-cytometry analysis for cell-surface screening demonstrated that the chondrogenic markers CD105, CD90, CD73, CD49f and CD44 are highly expressed on the surface of isolated ankle human chondrocytes and did not changed their expression during time in culture. On the other hand, the MSCs markers CD45 and CD34 were not detected and the lack of expression was maintained through the culture passages. The present results confirm successful optimization of the protocol for isolation of human chondrocytes from patients' ankles. Moreover, immunocytochemical analysis can be a useful technique for detecting the deposition of matrix, as well as distinguishing whether either of these cells are producing type I or type II collagen during 2D culture. As already 

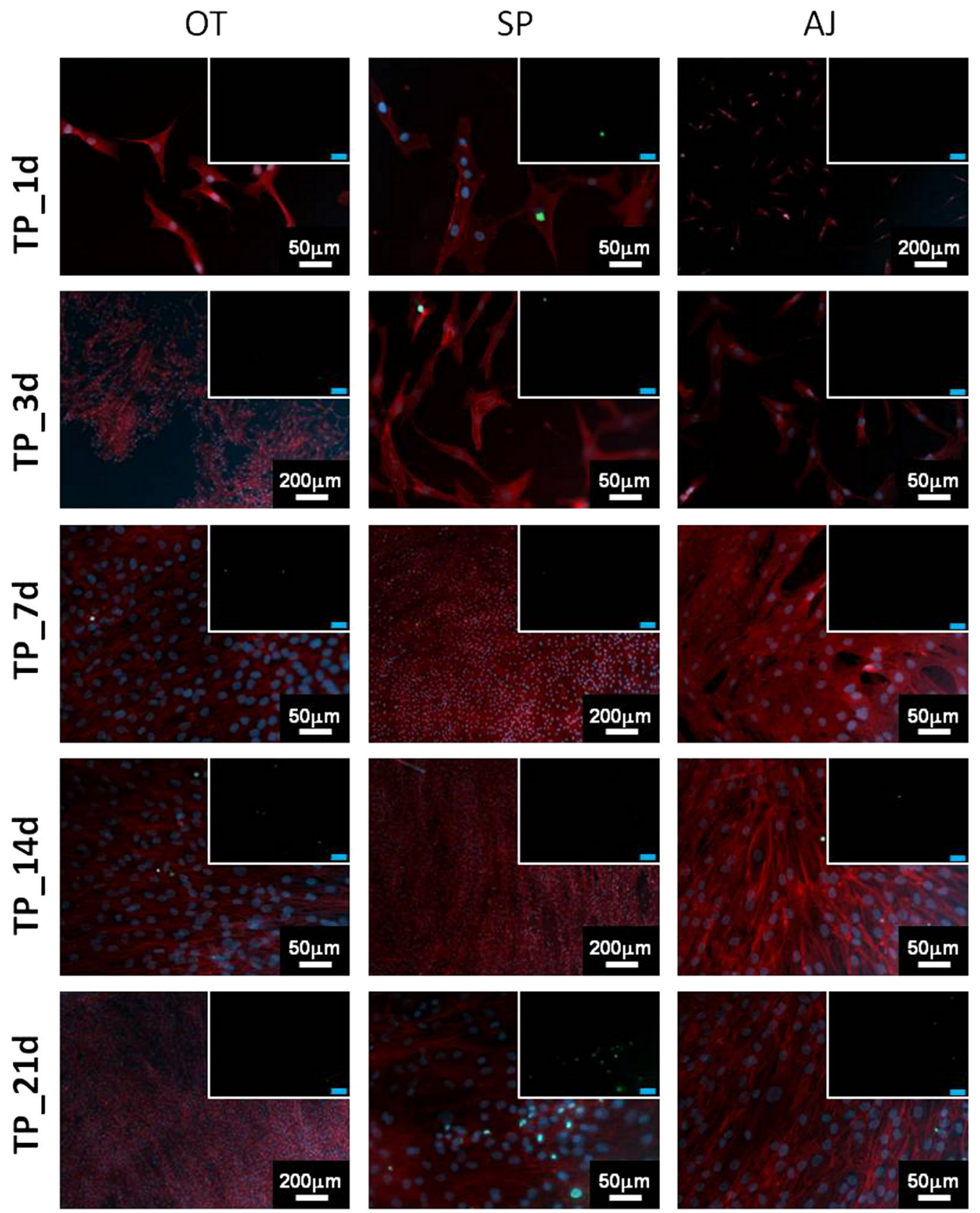

Figure 6. TUNEL assay imaging of cells isolated from ankle tissues and cultured in vitro for 1-21 days (d; small images); counterstaining images with phalloidin (cytoskeleton) and DAPI (nuclei) are also shown (large images); OT, os trigonum; SP, Stieda's process; AJ, ankle joint; TP, time point; blue scale bar $=50 \mu \mathrm{m}$

stated, the major compounds in hyaline-like cartilage extracellular matrix are type II collagen fibres and glycosaminoglycans. Further studies should be performed in order to evaluate matrix deposition using immunocytochemistry and gene expression by quantitative real-time reverse transcription analysis of relevant markers (e.g. glycosaminoglycans, type I and type II collagen).

It is important to bear in mind that the in vitro experimental design to characterize the post-expansion chondrogenic capacity of ankle chondrocytes does not directly allow us to predict the in vivo reparative/ regenerative ability of the cell types, i.e. if these cells were implanted in the talar lesions, no information is available at this stage to safely assess their biological performance.
Thus, subsequent studies are still required prior to in vivo use. Experiments comprising the culture of the isolated cells from ST or OT onto a 3D porous matrix in the presence of chondrogenic inducers, either in static or dynamic conditions, and in vivo studies using relevant models, are future research directions that need to be pursued as proof-of-concept.

Most of the clinical studies using MACI and ACI procedures have been performed on the knee (Correia et al., 2014), where chondrocytes are usually harvested from healthy cartilage on the ipsilateral side. It has been shown that ankle and knee chondrocytes have similar biosynthetic activities (Candrian et al., 2009). Despite requiring further clinical evaluation, it can be stated that the ankle 

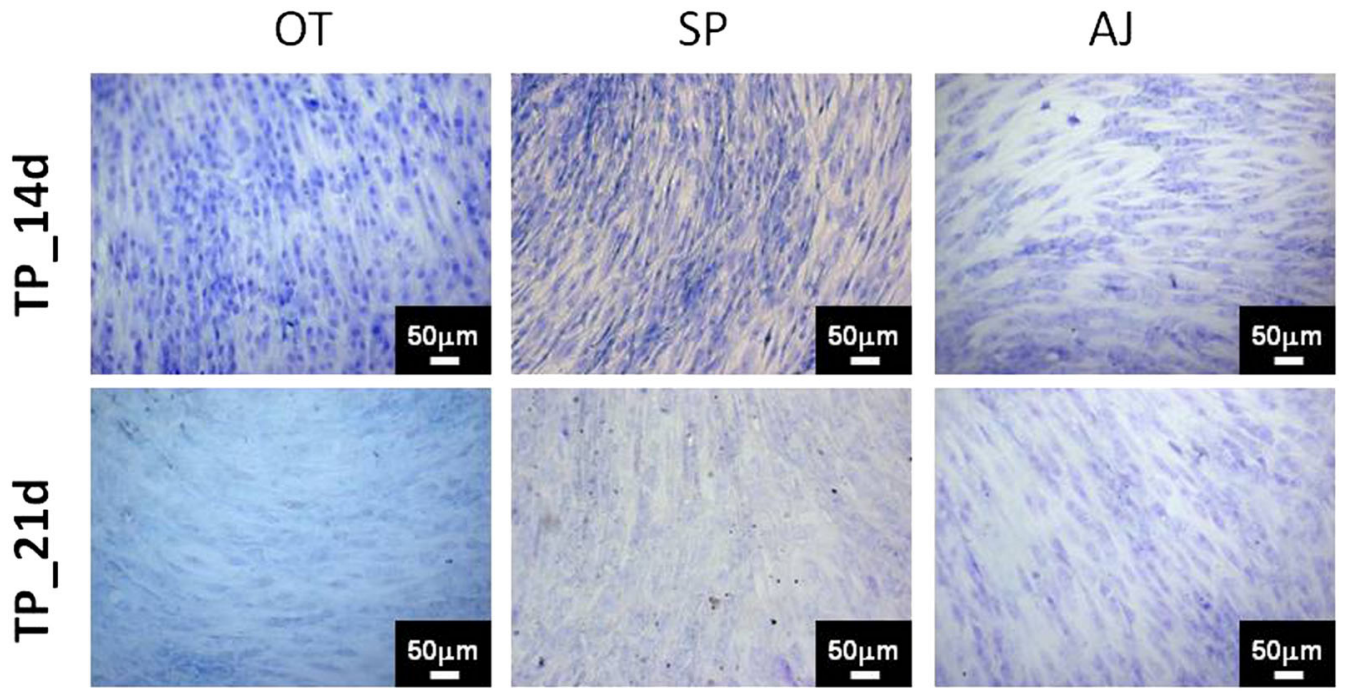

Figure 7. Collagen detection by toluidine blue staining on cells isolated from ankle tissues and cultured in vitro for 1-21 days (d). Although only TP_14d and TP_21d had shown positive staining, no relevant detection of collagen was observed throughout the other tested periods (i.e. TP_1d, TP_3d and TP_7d); OT, os trigonum; SP, Stieda's process; AJ, ankle joint; TP, time point

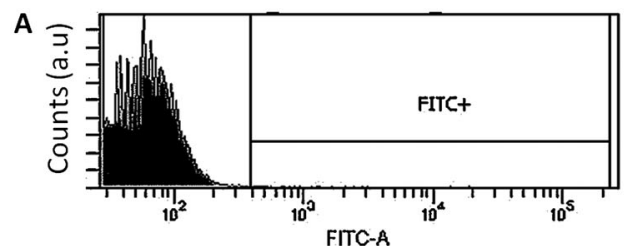

B
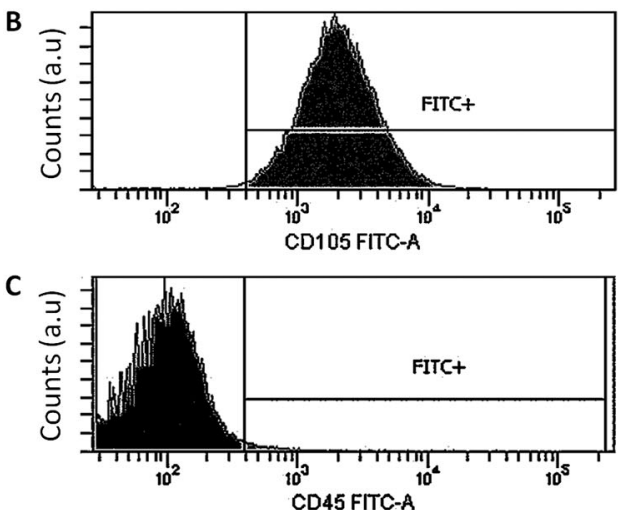

\begin{tabular}{|cc|c|}
\hline Cell Surface Markers & $\begin{array}{c}\text { P2-P3 (\%) } \\
\boldsymbol{X} \pm \boldsymbol{S} . \boldsymbol{D} .\end{array}$ \\
\hline \multirow{2}{*}{ Chondrogenic } & $\mathrm{CD} 105$ & $87.6 \pm 8.8$ \\
\cline { 2 - 3 } & $\mathrm{CD} 73$ & $90.8 \pm 8.2$ \\
\cline { 2 - 3 } & $\mathrm{CD} 49 \mathrm{f}$ & $85.3 \pm 6.2$ \\
\cline { 2 - 3 } & $\mathrm{CD} 44$ & $90.8 \pm 8.1$ \\
\hline MSCs & $\mathrm{CD} 45$ & $0.6 \pm 0.4$ \\
\hline & $\mathrm{CD} 34$ & $0.9 \pm 1.5$ \\
\hline
\end{tabular}

Figure 8. Phenotypic evaluation of cells isolated from ankle tissues by flow cytometry (FCM). Monolayer cultured cells were evaluated for the expression of the human chondrogenic surface markers CD105, CD90, CD73, CD49f and CD44 and the MSC markers CD45 and CD34. (A) FCM histogram for FITC fluorescence calibration showing the absence of autofluorescent expression of FITC by human chondrocytes. (B) FCM histogram showing the fluorescence intensity distribution of CD105-FITC-stained cells. (C) FCM histogram showing the fluorescence intensity distribution of CD45-FITC-stained cells. (D) Percentage of living chondrocytes expressing each analysed cell surface marker. Chondrocytes were obtained from three different donors and were analysed at passages 2 and 3 ( $n=6$ for each cell surface marker; $x \pm \mathrm{SD}$, mean \pm standard deviation)

can be a source of viable chondrocytes for a possible application in the treatment of cartilage lesions and OCDs in the knee joint.

\section{Conclusions}

In this study, viable chondrocytes were isolated and cultured from the posterior process of the talus (SP) and the os trigonum (OT). This study points out a new direction by setting up a suitable novel source of chondrocytes (and establishing the methods for isolation and cultivation in vitro) that can be potentially used in cell-based and tissue-engineering strategies for the treatment of cartilage and osteochondral lesions. One of the advantages of using this approach, which therefore emphasizes the importance of this study, is that the SP and OT tissues can be obtained by using minimally invasive surgical approaches, thus limiting the side-effects usually related with the classical approaches. The results obtained herein are promising, but further in vitro and in vivo 
preclinical investigations are required to determine the feasibility and safety of this approach prior to its use in clinics.

\section{Conflict of interest}

The authors declare no conflicts of interest.

\section{Acknowledgements}

The authors are grateful for funds provided by the Portuguese Foundation for Science and Technology (FCT) through the project OsteoCart (Grant No. PTDC/CTM-BPC/115977/2009), as well as the $\mathrm{PhD}$ scholarship provided to $\mathrm{R}$. F. Canadas (Grant No. SFRH/BD/92565/2013). The FCT distinction attributed to J. M. Oliveira under the Investigador FCT programme (Grant No. IF/00423/2012) is also greatly acknowledged. The authors are also grateful for funds provided by Fundación MAPFRE (Ayudas a la Investigación Ignacio H. de Larramendi, Prevención, Salud y Medio Ambiente, Spain) under the project 'Preventing the progression of the knee osteoarthritis: advanced therapies combining injectable hydrogels, autologous stem cells and PRP' (Grant No. BIL/13/SA/235). This study was also carried out with the support of Fundo Europeu de Desenvolvimento Regional (FEDER) through Programa Operacional do Norte through the project Articulate (Grant No. 23189).

\section{References}

Anders S, Goetz J, Schubert T, et al. 2012; Treatment of deep articular talus lesions by matrix associated autologous chondrocyte implantation - results at five years. Int Orthop 36: 2279-2285.

Aurich M, Bedi HS, Smith PJ, et al. 2011; Arthroscopic treatment of osteochondral lesions of the ankle with matrix-associated chondrocyte implantation. Am J Sports Med 39: 311-319.

Barbero A, Grogan SP, Mainil-Varlet P, et al. 2006; Expansion on specific substrates regulates the phenotype and differentiation capacity of human articular chondrocytes. J Cell Biochem 98: 1140-1149.

Baums MH, Heidrich G, Schultz W, et al. 2006; Autologous chondrocyte transplantation for treating cartilage defects of the talus. $J$ Bone Joint Surg Am 88: 303-308.

Benya PD, Shaffer JD 1982; Dedifferentiated chondrocytes re-express the differentiated collagen phenotype when culture in agarose gels. Cell 30: 215-224.

Bizarro AH 1921; On sesamoid and supernumerary bones of the limbs. $J$ Anat $\mathbf{5 5}$ 256-268.

Candrian C, Bonacina E, Frueh JA, et al. 2009; Intra-individual comparison of human ankle and knee chondrocytes in vitro: relevance for talar cartilage repair. Osteoarthr Cartilage 17: 489-496.

Candrian C, Miot S, Wolf F, et al. 2010 Are ankle chondrocytes from damaged fragments a suitable cell source for cartilage repair? Osteoarthr Cartilage 18: 1067-1076.

Chao W 2004; Os trigonum. Foot Ankle Clin 9: 787-796.

Correia SI, Pereira H, Silva-Correia J, et al. 2014; Current concepts: tissue engineering and regenerative medicine applications in the ankle joint. $J R$ Soc Interface 11: 20130784.

Garvican ER, Vaughan-Thomas A, Redmond C, et al. 2008; Chondrocytes harvested from osteochondritis dissecans cartilage are able to undergo limited in vitro chondrogenesis despite having perturbations of cell phenotype in vivo. J Orthop Res 26: 1133-1140.

Giannini S, Buda R, Grigolo B, et al. 2005; The detached osteochondral fragment as a source of cells for autologous chondrocyte implantation (ACI) in the ankle joint. Osteoarthr Cartilage 13: 601-607.

Giannini S, Buda R, Vannini F, et al. 2009, One-step bone marrow-derived cell transplantation in talar osteochondral lesions. Clin Orthop Relat Res 467: 3307-3320.

Giannini S, Buda R, Vannini F, et al. 2008; Arthroscopic autologous chondrocyte implantation in osteochondral lesions of the talus. Am J Sports Med 36: 873-880.

Giza E, Sullivan M, Ocel D, et al. 2010; Matrix-induced autologous chondrocyte implantation of talus articular defects. Foot Ankle Int 31: 747-753.

Gobbi A, Francisco RA, Lubowitz JH, et al. 2006; Osteochondral lesions of the talus: randomized controlled trial comparing chondroplasty, microfracture, and osteochondral autograft transplantation. Arthroscopy 22: 1085-1092.

ISO/EN10993-5 2009; Biological evaluation of medical devices. Part 5: Tests for in vitro cytotoxicity, Organization IS: Geneva, Switzerland.

Junqueira LC, Carneiro J, Wisse E 2007; Functionele Histologie, 11th edn.Elsevier: Maarssen, The Netherlands.

Kuroki K, Cook JL, Tomlinson JL, et al. 2002; In vitro characterization of chondrocytes isolated from naturally occurring osteochondrosis lesions of the humeral head of dogs. Am $J$ Vet Res 63: 186-193.

O'Driscoll SW 1998; The healing and regeneration of articular cartilage. $J$ Bone Joint Surg Am 80: 1795-1812.

Park CH, Kim SY, Kim JR, et al. 2013; Arthroscopic excision of a symptomatic os trigonum in a lateral decubitus position. Foot Ankle Int 34: 990-994.

Qiu YS, Shahgaldi BF, Revell WJ, et al. 2003 Observations of subchondral plate advancement during osteochondral repair: a histomorphometric and mechanical study in the rabbit femoral condyle. Osteoarthr Cartilage 11: 810-820.

Rathur S, Clifford P, Chapman C 2009; Posterior ankle impigemente: os trigonum syndrome. Am J Orthop 38: 252-253.

Reddy S, Pedowitz DI, Parekh SG, et al. 2007; The morbidity associated with osteochondral harvest from asymptomatic knees for the treatment of osteochondral lesions of the talus. Am J Sports Med 35: 80-85.
Russell JA, Kruse DW, Koutedakis Y, et al. 2010; Patho-anatomy of posterior ankle impingement in ballet dancers. Clin Anat 23: 613-621.

Schachter AK, Chen AL, Reddy PD, et al. 2005; Osteochondral lesions of the talus. J Am Acad Orthop Surg 13: 152-158.

Schneider TE, Karaikudi S 2009; Matrix-induced autologous chondrocyte implantation (MACI) grafting for osteochondral lesions of the talus. Foot Ankle Int $\mathbf{3 0}$ 810-814.

Tay AG, Farhadi J, Suetterli R, et al. 2004; Cell yield, proliferation, and postexpansion differentiation capacity of human ear, nasal and rib chondrocytes. Tissue Eng 10: 762-770.

Tey M, Monllau J, Centenera J, et al. 2007; Benefits of arthroscopic tuberculoplasty in posterior ankle impingement syndrome. Knee Surg Sports Traumatol Arthrosc 15: 1235-1239.

Valderrabano V, Leumann A, Rasch $\mathrm{H}$, et al. 2009; Knee-to-ankle mosaicplasty for the treatment of osteochondral lesions of the ankle joint. Am $J$ Sports Med 37: 105-111S.

Valderrabano V, Miska M, Leumann A, et al. 2013; Reconstruction of osteochondral lesions of the talus with autologous spongiosa grafts and autologous matrixinduced chondrogenesis. Am J Sports Med 41: 519-527.

van Dijk CN, Reilingh ML, Zengerink M, et al. 2010; Osteochondral defects in the ankle: why painful? Knee Surg Sports Traumatol Arthrosc 18: 570-580.

van Dijk CN, Scholten PE, Krips R 2000; A two-portal endoscopic approach for diagnosis and treatment of posterior ankle pathology. Arthroscopy 16: 871-876.

Vannini F, Filardo G, Kon E, et al. 2013; Scaffolds for cartilage repair of the ankle joint: the impact on surgical practice. Foot Ankle Surg 19: 2-8.

Vasiliadis HS, Wasiak J 2010; Autologous chondrocyte implantation for full-thickness articular cartilage defects of the knee. Cochrane Database Syst Rev: CD003323, DOI: 10.1002/14651858.CD003323.pub3.

Zengerink M, Struijs PA, Tol JL, et al. 2010; Treatment of osteochondral lesions of the talus: a systematic review. Knee Surg Sports Traumatol Arthrosc 18: 238-246. 


\section{Supporting information}

Additional supporting information may be found in the online version of this article at the publisher's web-site:

Figure S1. Scheme of the human ankle tissues, identifying the location of Stieda's process, the os trigonum (OT) and SP after arthroscopic excision

Figure S2. Fluorescence microscopy images of Stieda's process (SP1) after immunohistochemical localization of type I collagen, control sample without staining or antibodies and sample treated with type I collagen primary antibody and AlexaFluor 488 secondary antibody

Figure S3. Cartilage cell counting within each sample

Table S1. Primary and fluorescent-labelled secondary antibodies used in immunohistochemical analysis Supplementary Figures Captions 